by Ralph Lee Scott

\title{
Computer Housekeeping
}

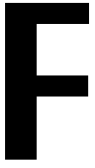

all is a good time to do housekeeping, both around the house and with your computer. Some basic things you can do to improve your computer's performance are deleting temporary Internet files, removing old unused documents, using system-cleanup tools, and cleaning up your desktop. Making backups and system-restore disks are also important aspects of good computer housekeeping.

Cleaning temporary Internet files from your machine will improve performance and at the same time protect your computer from cookies installed by outside parties. To clean temporary files in Internet Explorer, go to Tools: Internet Options: General: "Delete Cookies": "Delete Files": "Clear History." In Netscape, go to Edit: Preferences: Advanced: Cache: "Clear Memory Cache": "Clear Disk Cache." Keep temporary Internet files to a minimum. When you delete these temporary files, you will remove any password that you have stored under the "remember this password" option. This is actually a good idea because it prevents hackers from searching your hard disk for passwords.

Another basic piece of protection is a restore disk. For PCs with Windows 98, go to Start: Programs: Accessories: System Tools: Emergency Repair Disk. For PCs with Windows 2000, open the Backup menu and selecting from the Tools pull-down menu: Create an Emergency Repair Disk. For Apple computers, you can just reinstall the operating system software and your other files will be fine.

Another important part of fall computer housekeeping is to check and backup your document files. Delete unused and outdated documents along with any drafts no longer needed. Do this for all data files such as e-mail, word processing files, spreadsheets, databases, and Web pages. Make backup copies of important files now and do it often in the future. You can save small files to floppies, but for larger files burn a CD or use a Zip disk or tape backup. To make a backup in Windows 2000, go to Start: Programs: Accessories: Systems Tools: Backup. Follow the directions for making the backup. For Apple computers, you can purchase backup software or just drag and copy the files to the additional backup media. Be sure to save your bookmarks, address books, and other necessary files on a floppy. While you are at it, clean up your desktop by removing icons that you will never use again.

Using system-cleanup tools such as defragmenters and disk clean-up tools can help keep your files in order. In Windows 2000, you can find clean-up tools under Start: Programs: Accessories: Systems Tools: Disk Defragmenter: Disk Cleanup. Apple computer users can find similar type programs under "Disk First Aid." You can also save space by compressing your data using a program like WinZip. While you are compressing your files, check the Event Viewer to note any problems your computer is having. You can find the Event Viewer on Windows 2000 machines by going to Start: Settings: Control Panel: Administrative Tools: Event Viewer. Here you will find the Application log, the Security log, and the System log. These logs track most abnormal operations of your hardware and software and provide information useful for troubleshooting problems.

Having effective virus-scanning software is very important to users of e-mail and the Internet. I check each morning for virus file updates. Remember to set your virus-scanning software to the highest level of security. In Norton software, this highest level of security is reached by clicking on the tab labeled "Heuristics." Here you can change the level of virus-scanning security from high to low. Most anti-virus software will let you set what happens to files with viruses in them at the time of checking. Configure the software to first delete the virus, then, as a second choice, quarantine the infection files. Often, the default choice as to what happens is to quarantine, rather than delete, allowing many infected files to remain on your computer. Always delete the infected files, if possible. Scan your hard drives at least once a week and check all floppies each time you insert them.

Making a backup not only of data files, but also of system and application software will give you peace of mind. It is also a good practice when you do your computer housekeeping to make a new backup at that time so that you will have a complete backup of everything that can quickly be reinstalled should hardware or software fail. Backups should be made at least weekly for critical files. You might also think about off-site storage for mission-critical files.

Just remember that the fall and spring are great times to clean up your home-and don't forget your computer! 